\title{
Características clínicas y anatomopatológicas en mola hidatiforme*
}

\author{
JOSÉ PACHECO D ${ }^{1,2}$, JAIME ALEGRE ${ }^{3}$, GISSELA PANIAGUA ${ }^{1}$ \\ ${ }^{1}$ Instituto de Patología de la UNMSM. ${ }^{2}$ Instituto de Medicina Legal. ${ }^{3}$ Hospital Nacional Dos de Mayo.
}

\begin{abstract}
RESUMEN
OBJETIVO: Determinar los hallazgos clínicos y anatomopatológicos (AP) en los casos de mola hidatiforme (MH). DISEÑO: Estudio descriptivo retrospectivo. MATERIAL Y MÉTODOS: Se revisó todos los casos de embarazo molar (EM) en gestantes del Hospital Nacional "Dos de Mayo" (HNDM) entre los años 1998 y 2001. RESULTADOS: De 24 diagnósticos de MH, la edad promedio fue 26,4 años $\pm 6,4$ y la edad gestacional de 13,1 semanas $\pm 3,3$. Como antecedentes obstétricos, sólo se encontró 1 y 3 casos de hiperemesis gravídica y de abortos previos, respectivamente; 18 gestantes fueron multíparas. Los síntomas al ingreso fueron dolor hipogástrico $(75 \%)$, náuseas y vómitos $(29,2 \%)$, disuria $(20,8 \%)$ y cefalea $(8,3 \%)$; y los signos clínicos, ausencia de feto (100\%), sangrado vaginal $(91,7 \%)$, útero blando $(45,8 \%)$, altura uterina discordante $(33,3 \%)$ y expulsión de vesículas $(25 \%)$. La ecografía concluyó en MH con ausencia fetal $(100 \%)$, ovarios aumentados de volumen $(29,2 \%)$ y quistes tecaluteínicos $(25 \%)$. El valor promedio de $\beta$ hCG fue $18167 \mathrm{UI} / \mathrm{L}$. Se utilizó misoprostol transvaginal como tratamiento previo al legrado uterino en $37,5 \%$. Los hallazgos AP fueron hiperplasia moderada del epitelio coriónico (62,5\%), estroma avascular (50\%) y vellosidades edematosas $(20 \%)$. CONCLUSION: Los hallazgos evidenciados en el presente estudio son características infrecuentes a lo descrito en la literatura médica de esta patología.
\end{abstract}

Palabras clave : Mola hidatiforme; complicaciones del embarazo; neoplasmas uterinos.

\section{HYDATIDIFORM MOLE CLINICAL AND PATHOLOGICAL CHARACTERISTICS ABSTRACT}

OBJECTIVE: To determine clinical and pathological findings in hydatidiform mole. DESIGN: Descriptive retrospective study. MATERIAL AND METHODS: All cases de molar pregnancy attended at Dos de Mayo National Hospital (HNDM) between 1998 and 2001 were reviewed. RESULTS: Twenty-four cases of hydatidiform mole were attended; mean age was 26,4 years $\pm 6,4$ and gestational age 13,1 weeks $\pm 3,3$. Past history only showed 1 and 3 cases of hyperemesis gravidarum and previous abortions, respectively; 18 pregnant women were multiparae. Symptoms on admission were hypogastric pain (75\%), nausea and vomiting $(29,2 \%)$, dysuria $(20,8 \%)$ and headache $(8,3 \%)$; and clinical sigsn, fetal absence (100\%), vaginal bleeding $(91,7 \%)$, soft uterus $(45,8 \%)$, discordant uterine height $(33,3 \%)$ and expulsion of vesicles $(25 \%)$. Ultrasound diagnosis was hydatidiform mole with fetal absence (100\%), ovarian enlargement $(29,2 \%)$ and tecaluteinic cysts (25\%). Average $\beta$ hCG was $18167 \mathrm{UI} / \mathrm{L}$. Transvaginal misoprostol was used previouly to dilatation and curettage in 37,5\%. Pathology findings were corionic epitheliu moderate hyperplasia $(62,5 \%)$, avascular estroma $(50 \%)$ and edematous villi (20\%). CONCLUSION: Our findings denote infrequent characteristics to the literature description on this pathology.

Key words: Hydatidiform mole; pregnancy complications; uterine neoplasms.

* El resumen del presente trabajo fue publicado en Anales de la Facultad de Medicina, Volumen 63, Suplemento 2002.

Correspondencia:

Dr. José Luis Pacheco De La Cruz.

Instituto de Patología, Facultad de Medicina - UNMSM.

Av. Grau 755. Lima 1, Perú

E-mail: jpachecod@terra.com 


\section{INTRODUCCIÓN}

La enfermedad trofoblástica gestacional es una entidad con aspectos aún no claros y precisos. Su forma de valoración es importante para prevenir y detectar la enfermedad $\left({ }^{1,2}\right)$. Clínicamente abarca alteraciones benignas -como la mola hidatiforme- y malignas, como el coriocarcinoma, siendo una entidad intermedia, el tumor trofoblástico de sitio placentario $\left(^{3}\right)$.

La mola hidatiforme se caracteriza por una tumefacción quística de las vellosidades placentarias, acompañada de una proliferación trofoblástica variable. La razón más importante para diagnosticar correctamente las molas verdaderas es que éstas son las precursoras más frecuentes del coriocarcinoma $\left({ }^{4}\right)$.

La mayoría de autores señalan una mayor incidencia de mola en mujeres por encima de los 40 y 50 años de edad $\left({ }^{3,5-7}\right)$. Otros sugieren que las mujeres menores de 20 años de edad tienen un mayor riesgo $\left({ }^{8}\right)$. Existen varios casos reportados de embarazos molares en una misma mujer, no siendo esto tan infrecuente $\left(^{9}\right)$.

Por razones mal conocidas, la incidencia varía considerablemente en distintas regiones del mundo: 1 por cada 1000 embarazos en Estados Unidos, pero 10 por cada 1000 en Indonesia $\left({ }^{10}\right)$. En el Perú, se encuentra frecuencias diferentes, como de 1 en 213 y 241 para los Hospitales Arzobispo Loayza y María Auxiliadora, respectivamente, así como de 1 en 476, 488 y 665 para el Instituto Materno Perinatal, Hospital Cayetano Heredia de Piura y San Bartolomé $\left({ }^{7,11}\right)$.

La mayor parte de las pacientes son embarazadas de cuatro a cinco meses de gestación $\left({ }^{12}\right)$. Clásicamente presentan hemorragia de la primera mitad del embarazo, hiperemesis severa y toxemia precoz. La ecografía puede permitir el diagnóstico antes de que se inicien los síntomas, que para la mola completa sucede a las 6 a 8 semanas y para la parcial entre las 16 a 18 semanas $\left({ }^{13}\right)$. La hemorragia es el síntoma más constante, encontrándose en 90 a $97 \%$ de los casos $\left(^{5,14-16}\right)$. El hallazgo de un útero de mayor tamaño al que corresponde por amenorrea ha sido encontrado en 38 a $51 \%\left({ }^{14,17}\right)$. La hiperemesis puede ocurrir de 20 a $26 \%$, según algunos estudios $\left({ }^{18}\right)$. La toxemia precoz se presenta, paradójicamente, en momento tardío en el embarazo molar, teniendo una frecuencia de $27 \%\left({ }^{14,16}\right)$ y de $12 \%$, según la zona geográfica. Existe la formación de quistes tecaluteínicos por una respuesta exagerada de los ovarios a niveles elevados de hCG, los cuales son generalmente bilaterales y con una frecuencia entre 15 a $25 \%(3,5,13)$. Es considerablemente mayor la frecuencia de pacientes con mola que tienen pruebas de laboratorio de función tiroidea alterada e incrementada $\left({ }^{19,20}\right)$.

Actualmente, se puede distinguir dos clases de mola no invasora, la completa y la parcial, de acuerdo con los estudios histológicos, citogenéticos y de citometría de flujo $\left.{ }^{(21}\right)$. Ambos tipos de mola también desarrollan otras características histológicas que subrayan su diferencia $\left({ }^{22}\right)$. En la mola completa (o clásica), todas o la mayoría de las vellosidades están edematosas, existiendo una hiperplasia trofoblástica difusa; no hay desarrollo embrionario; por lo tanto, las molas completas no contienen partes fetales. En las molas parciales hay edema en algunas vellosidades y la proliferación trofoblástica es focal; el embrión es viable durante unas semanas, por lo que puede encontrarse partes fetales cuando se produce el aborto de mola así formada.

Para algunos autores, la clasificación histológica en diversos grados no tiene significado pronóstico $\left({ }^{23}\right)$; por tanto, todas las molas deben ser cuidadosamente vigiladas mediante la determinación seriada de los niveles de hCG. Una vez hecho el diagnóstico, debe extirparse la mola mediante un legrado uterino completo. En las pacientes que no desean tener más hijos, puede realizarse una histerectomía.

Según numerosos estudios, está claro que 80$90 \%$ de estas molas son benignas y no vuelven a dar problemas. Un $10 \%$ evoluciona hacia molas invasoras y $2,5 \%$ hacia un coriocarcinoma $\left({ }^{24}\right)$. 
Siendo una patología obstétrica relativamente poco frecuente, pero con una alta posibilidad de ser identificada durante el control prenatal, analizaremos y describiremos las manifestaciones clínicas encontradas en los casos de mola hidatiforme en una institución, con el fin de que esta patología no sea una sorpresa para el examinador. A su vez, identificaremos los hallazgos anatomopatológicos encontrados en cada tipo de mola, con el propósito de resaltar la importancia que tiene el envío de muestras post legrado y su relación con la progresión de patologías invasivas o malignas.

\section{MATERIAL Y MÉTODOS}

Se realizó un estudio descriptivo restrospectivo en el que se revisó todos los reportes ecográficos de las pacientes que habían ingresado con diagnóstico clínico de embarazo molar al Departamento de Gineco-Obstetricia del Hospital Nacional Dos de Mayo, entre 1998 y 2001. Se procedió a ubicar el número de historias clínicas para su respectiva revisión.

Se consideró las siguientes variables: edad de la paciente, tiempo de amenorrea, antecedentes obstétricos, paridad, síntomas de ingreso, signos clínicos durante la exploración física, hallazgos ecográficos, indicaciones terapéuticas, resultado del informe anatomopatológico, entre otras. Se elaboró una hoja de recolección de datos para cada una de las historias clínicas, siendo procesados con el programa estadístico de Microsoft Excel 2000. Algunas láminas histopatológicas fueron revisadas nuevamente para la identificación y comprobación de los hallazgos anteriormente descritos.

\section{RESULTADOS}

Se ubicó 24 casos de embarazo molar, los cuales fueron diagnosticados clínicamente como mola hidatiforme, tanto en el servicio de emergencia como en consultorios externos (19 y 5 casos, respectivamente). De éstos, 3 casos pre- sentaron el diagnóstico clínico inicial de aborto incompleto.

La edad promedio de las gestantes fue 26,4 años $\pm 6,4$ (intervalos de 15 a 40 años). La edad gestacional (EG) promedio fue calculada en 13,1 semanas $\pm 3,3$.

En la Figura 1 se muestra la paridad de las gestantes. Se encontró una mayor frecuencia en pacientes multíparas (18 casos).

Se buscó antecedentes obstétricos, reportándose sólo un caso de hiperemesis gravídica previa en una paciente multípara y 3 casos de abortos previos en nulíparas. No se evidenció algún caso de embarazo molar previo ni antecedente de enfermedad tiroidea.

Los síntomas más frecuentes que se evidenció al ingreso fueron el dolor abdominal hipogástrico y la presencia de náuseas y vómitos (Tabla 1). No se presentaron síntomas de hipertiroidismo, como temblor, adelgazamiento, entre otros. De igual manera, se determinó que los signos más frecuentes durante la exploración física obstétrica fueron la ausencia de signos fetales (latidos cardíacos) y la presencia de sangrado vaginal, signo que comúnmente alerta a la paciente. Como se aprecia en la Ta-

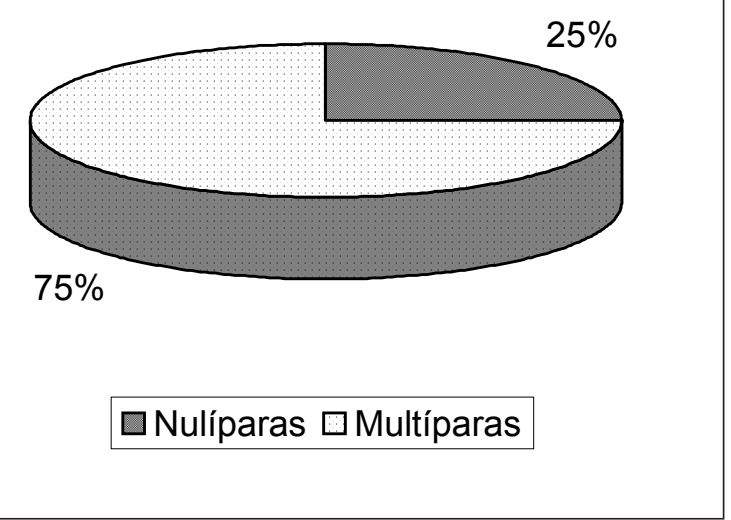

Figura 1.- Paridad en los casos de mola hidatiforme. 
Tabla 1.- Síntomas de mola hidatiforme

\begin{tabular}{lrr}
\hline Síntoma & $\mathrm{n}$ & $\%$ \\
\hline Dolor hipogástrico & 18 & 75,0 \\
Náuseas y vómitos & 7 & 29,2 \\
Disuria & 5 & 20,8 \\
Cefalea & 2 & 8,3 \\
\hline
\end{tabular}

bla 2, no se evidenció signos de hipertensión arterial, edema y demás manifestaciones de la preeclampsia.

Tabla 2.- Hallazgos del examen físico en mola hidatiforme.

\begin{tabular}{lcr}
\hline Signo & $\mathrm{n}$ & \multicolumn{1}{c}{$\%$} \\
\hline Ausencia de signos fetales & 24 & 100,0 \\
Sangrado vaginal & 22 & 91,7 \\
Útero blando & 11 & 45,8 \\
$\begin{array}{l}\text { Altura uterina discordante o } \\
\text { "signo del acordeón" }\end{array}$ & 8 & 33,3 \\
$\begin{array}{l}\text { Expulsión de vesículas } \\
\text { semejantes a "uvas" }\end{array}$ & 6 & 25,0 \\
\hline
\end{tabular}

Se realizó ecografías abdominales y vaginales en 14 y 10 de los casos, respectivamente (Figura 2).
Los hallazgos ecográficos principales fueron ausencia de partes fetales y presencia de vellosidades vesiculares tipo "tormenta de nieve" (Figura 3 y Tabla 3).

Tabla 3.- Hallazgos ecográficos en mola hidatiforme.

\begin{tabular}{lrr}
\hline Hallazgo & $\mathrm{n}$ & \multicolumn{1}{c}{$\%$} \\
\hline Ausencia fetal & 24 & 100,0 \\
Vellosidades vesiculares & 24 & 100,0 \\
Ovarios aumentados de volumen & 7 & 29,2 \\
Quistes tecaluteínicos & 6 & 25 \\
\hline
\end{tabular}

Otro procedimiento diagnóstico evaluado fue la cuantificación del dosaje sérico de $\beta$ hCG. Este cálculo promedio fue de $18167 \mathrm{UI} / \mathrm{L}$ (valor máximo de 160 000). También se analizó los resultados del dosaje de hemoglobina al momento de ingreso, presentando un promedio de 9,32 $\mathrm{g} \%$ (intervalos de 3,4 a 12,4).

Respecto a las medidas terapéuticas, los tratamientos previos al legrado uterino se basaron en misoprostol transvaginal ( 9 casos) y oxitocina EV (12 casos), principalmente. La ergotamina VO fue el medicamento que más frecuentemen-

Figura 3.- Mola hidatiforme. Se evidencia múltiples imágenes vesiculares y quísticas en cavidad uterina. Imagen típica de "tormenta de nieve" o "panal de abejas". 
te se utilizó luego de dicho procedimiento (21 casos). Se reportó un caso de histerectomía abdominal total y salpingooforectomía bilateral.

El $100 \%$ de los casos evolucionó favorablemente luego del tratamiento indicado, presentándose un tiempo promedio de estancia hospitalaria de 4,3 días $\pm 2,6$.

Las descripciones de los reportes anatomopatológicos de las muestras extraídas del legrado uterino fueron clasificadas en la Tabla 4. Se ubicó los resultados de 24 casos. De éstos, todos tuvieron hallazgos compatibles con mola hidatiforme de tipo parcial (Figura 4).

Tabla 4.- Hallazgos anatomopatológicos en mola hidatiforme.

\begin{tabular}{lrc}
\hline Hallazgo & $\mathrm{n}$ & $\%$ \\
\hline Hiperplasia moderada & & \\
del epitelio coriónico & 15 & 62,5 \\
Estroma avascular & 12 & 50,0 \\
Vellosidades edematosas & 5 & 20,8 \\
\hline
\end{tabular}

\section{DISCUSIÓN}

La mola hidatiforme es una patología que no debe sorprender al clínico en el momento de su identificación. Para esto, se planteó el análisis del enfoque clínico, con el propósito de llegar al diagnóstico antes del estudio anatomopatológico.

En nuestra revisión, las características clínicas coinciden con las señaladas en la literatura médica. Sin embargo, reportamos una presentación de mola hidatiforme en mujeres alrededor de los 26 años, aproximadamente. Este es uno de los aspectos que muy pocas veces ha sido reportado, pues como se sabe, la mayor incidencia se presenta en gestantes mayores de 40 años de edad $\left({ }^{3,5-7}\right)$. A la vez, también existen reportes frecuentes en mujeres adolescentes $\left({ }^{8}\right)$.

También se ha evidenciado la ausencia de antecedentes obstétricos que muy frecuentemente se relacionan con esta patología. Entre éstos, reportamos la ausencia de embarazo molar previo y de enfermedad hipertiroidea. Así mismo, no hemos descrito algún signo que nos pueda sugerir la existencia de enfermedad hipertensiva del embarazo.

Es muy conocido que la enfermedad trofoblástica gestacional constituye una causa muy importante en las hemorragias del primer trimestre. Sin embargo, la evolución habitual de la mola vesicular es su expulsión espontánea alrededor del $4^{\circ}$ ó $5^{\circ}$ mes de gestación $\left({ }^{12}\right)$. Nuestro análisis revela la aparición de los signos de mola hidatiforme en gestantes antes de las 10 semanas de edad gestacional.

Cabe resaltar que una de las descripciones más importantes de nuestra revisión es la falta de identificación de partes embrionarias o fetales. Esto hizo suponer que ecográficamente se trataría de una mola hidatiforme completa; pero, como hemos visto, los hallazgos anatomopatológicos concluyeron en mola hidatiforme parcial. Tal conclusión debe sugerir que siempre se deberá enviar las muestras del legrado uterino al respectivo estudio patológico, pues como sabemos, está identificación también descartará la existencia de alguna neoplasia maligna.

Figura 4.- Mola hidatiforme parcial. Se observa escaso edema del epitelio coriónico. Hay vasos en las vellosidades con hiperplasia del estroma. 


\section{BIBLIOGRÁFÍA}

1. Mitchell MF, Scholtenfeld D, Hong WK. Prevención del cancer ginecológico. Clin Ginecol Obstet Mex 1996; 2: 500.

2. Paiva de Alvarez S, Zapata L, Santerini R, Pérez C. Mola hidatiforme: criterios diagnósticos más resaltantes. Rev Obstet Ginecol Venez 1989; 49(1): 13.

3. Copeland L, Jarrel J, McGregor J. Textbook of Gynecology. 1993.

4. Rodline RW, Abdul-Karim FW. Pathology of gestacional trophoblastic disease. Semin Oncol 1995; 22: 96.

5. Beck WW. Gestational trophoblastic disease. Obstetric and Gynecology. The National Medical Series for Independent Study. USA: Harwal Publishing. 1993.

6. Buckley JD. The epidemiology of molar pregnancy and choriocarcinoma. Clin Obstet Gynecol 1984; 27: 153.

7. Jefferson L, Vicuña R, Rechkemer A. Factores de riesgo en la enfermedad trofoblástica gestacional: estudio casocontrol. Ginecol Obstet (Perú) 1994; 40: 68.

8. Slocumb JC, Lund CJ. Incidence of trophoblastic disease: increased rate in youngest age group. Am J Obstet Gynecol 1969; 104: 421.

9. Federscheinder FM, Goldstein DP, Berkowitz RS, Marean AR, Berstein MR. Natural history of recurrent molar pregnancy. Obstet Gynecol 1980; 55: 457.

10. Bracken MB, Brinton LA, Hayashi K. Epidemiology of hydatidiform mole and choriocarcinoma. Epidemiol Rev 1984; 6: 52

11. Rodríguez-Frías E, Mendoza R, León F. Enfermedad del trofoblasto en el Hospital Regional Cayetano Heredia de Piura. Acta Médica Peruana 1985; 12: 35.

12. Robbins CK. Patología Estructural y Funcional. McGrawHill-Interamericana de España. S.A. Madrid. Cap 24:1130. $6^{\circ}$ Edición. 2000

13. Kohorn E. Embarazo molar: presentación y diagnóstico. Clin Obstet Gynecol NA 1984; 1: 235.
14. Berkowitz RS, Cramer DW, Bernstein MR, Cassells S, Driscoll SG, Goldstein DP. Risk factors for complete molar pregnancy from a case control study. Am J Obstet Gynecol 1985; 152: 1016.

15. Curry SL, Hammond CB, Tyrey L, Creasman WT, Parker RT. Hydatiform mole: diagnosis, management and long-term follow up of 347 patients. Obstet Gynecol 1975; 45: 1.

16. Goldstein DP, Berkowitz RS. Gestational trophoblastic neoplasms. En: Major problems in Obstetrics and Gynecology. Vol 14. Philadelphia: W.B. Saunders. 1983.

17. Hayashi K, Bracken MB, Freeman DH, Hellenbrand K. Hydatiform mole in the United States (1970-1977): a statistical and theoretical analysis. Am J Epidemiol 1982; 89: 258.

18. Jacobs PA, Hunt PA, Matsumura JS, Wilson CC, Szulman AE. Complete and partial hydatidiform mole in Hawaii: cytogenetics, morphology and epidemiology. Br J Obstet Gynecol 1982; 89: 258.

19. Dowling JT, Ingbar SH, Freinkel N. Iodine metabolism in hydatidiform mole and choriocarcinoma. J Clin Endocrinol Metab 1960; 20: 1.

20. Herschmann JJM, Higgings HP. Hydatidiform mole: a cause of clinical hyperthyroidism. N Engl J Med 1971; 284: 573.

21. Lage JM. Gestational trophoblastic tumors, refining histologic diagnosis by using DNA flow and image cytometry. Curr Opin Obstet Gynecol 1994; 6: 359.

22. Szulman AE, Surti U. The syndromes of hydatidiform moles: II. Morphologic evolution of the complete and partial mole. Am J Obstet Gynecol 1978; 132: 20.

23. Genest DR, Laborde O, Berkowitz RS, Goldstein DP, Bernstein MR, Lage J. A clinico-pathologic study of 153 cases of complete hydatidiform mole (1980-1990) : histologic grade lacks prognostic significance. Obstet Gynecol 1991; 78: 402.

24. Lurain JR, Brewer JI, Torok EE, Halpern B. Natural history of hydatidiform mole after primary evacuation. Am J Obstet Gynecol 1983; 145: 591. 